RAL Report

RAL-95-034

\title{
Monte Carlo Studies of Electron Avalanches in Microdosimetric Proportional Counters
}

H Prưchova and B Franĕk

March 1995 
DRAL is part of the Engineering and Physical Sciences Research Council

The Engineering and Physical Sciences Research Council does not accept any responsibility for loss or damage arising from the use of information contained in any of its reports or in any communication about its tests or investigations 
RAL-95-034

April 1995

\title{
Monte Carlo Studies of Electron Avalanches in Microdosimetric Proportional Counters
}

\author{
Helena Průchová* \\ Czech Technical University \\ Faculty of Nuclear Sciences and Physical Engineering \\ Department of Dosimetry and Application of Ionizing Radiation \\ Břehova 7, 11519 Praha 1, Czech Republic \\ Bohumil Franěk \\ Rutherford Appleton Laboratory \\ Chilton, Didcot, Oxon, OX11 0QX, United Kingdom
}

\begin{abstract}
We present preliminary results from a study of electron avalanches in a low pressure cylindrical microdosimetric counter. The technique is based on the Monte Carlo simulation of the motion of electrons in electric field and their interactions with the molecules of gas. All the electrons are traced simultaneously in time and their coordinates and velocities are recorded. It lets us study all temporal and space aspects of the electron avalanche development. In this work we have concentrated on a study of the dependence of gain, radial extent and time extent of the avalanche on the gas pressure. We explain our results by the existence of seeding region in the avalanche where a relatively small number of secondary electrons are created. Each of these electrons gives subsequently rise to a partial avalanche in the proper avalanche region.
\end{abstract}

(To be submitted to Nuclear Instruments and Methods)

*partially supported by Commission of European Community (contract FI3P-CT920045) 



\section{Introduction}

The topic of microdosimetry is related to the measurements of absorbed energy event distributions in volumes of tissue whose dimensions are equal or smaller than $1 \mu \mathrm{m}$. They are simulated by gas cavities filled with a tissue equivalent mixture of methane or propane based gas at low pressure. Tissue equivalent proportional counters (TEPC) are ordinarily constructed as small gas chambers of cylindrical or almost spherical shape. The walls are made from plastic (mainly Shonka plastic A-150) and are, at the same time, used as the cathode due to the plastic's conductivity. The anode is a thin wire located in the middle of the cylinder. The sensitive volume is filled with methane or propane gas at very low pressure in order to simulate small volumes of tissue. The working regime of these counters is already near the operational limit of gaseous detectors as had been reported by many authors as a result of their experimental work. In order to improve the design of these counters and possibly to extend their operational limit it would obviously be useful to have a theoretical tool that would allow to study and to help to understand the motion of electrons and ions inside these counters. There were a few attempts to develop such a tool, notably by Segur at al. [1] using the Boltzman equation. Because of the rapidly changing electric field in the vicinity of the anode it has been however difficult to find well proven numerical method for it's solution. Also, the studies have been focused on the dependence of the gas amplification on the gas pressure and applied voltage, not including a discussion of the spatial and temporal development of the electron avalanche.

In recent years there have been several attempts of Monte Carlo modelling of electron avalanches in electric field (e.g. $[2,3])$. These calculations were done for detectors used in high energy physics experiments. These detectors work normally under atmospheric pressure of gas. The reduced intensity of the electric field on the anode

surface,$\frac{E}{p}$, is therefore at least ten times smaller than in the case of microdosimetric counters.

We have developed a Monte Carlo technique suitable for the simulation of the electron avalanches also in microdosimetric counters. This allows us to visualize and to understand what is happening around the anode wire under the extreme conditions of high electric fields and low gas pressures. In this way, since all the electrons are traced simultaneously in time and their coordinates and velocities are recorded, all aspects of the electron avalanche development can be studied. Preliminary results from this 
study are presented in this paper.

\section{The method of Monte Carlo simulation}

The program starts from a given number of electrons deposited at chosen space points at time $t=0$. These electrons are then simultaneously traced in the cylindrical electric field in given time steps of equal length $\Delta t_{\mathrm{smp}}$ (sampling step). At the end of every $\Delta t_{\mathrm{smp}}$ the information about all the existing electrons at that time is recorded. Therefore the choice of this time step depends on how detailed temporal picture is required. It is usually of the order of a few psecs.

In each time interval $\left(t, t+\Delta t_{\mathrm{smp}}\right)$ every electron existing at time $t$ is independently traced from time $t$ to time $t+\Delta t_{\mathrm{smp}}$ as follows.

The electron is traced in small time steps $\Delta t_{\text {elm }}$ (elementary step) which are a fraction of $\Delta t_{\text {smp. }}$. The magnitude of this step is being recalculated from the electron's velocity and the electric field vector at the electron's current position. It is chosen in such a way that both, the electron's velocity and also the electric field vector at the new position change very little. In addition it is required that the electron travels only a fraction of it's mean free path, calculated from the electron-molecule cross-sections at the given energy, during the time interval $\Delta t_{\text {elm }}$. In each $\Delta t_{\text {elm }}$ it is decided whether there is a collision between the electron and a gas molecule by generating a random number and the interaction process is chosen according to the corresponding cross section tables. If there was no collision, electron is moved in the electric field in time $\Delta t_{\mathrm{elm}}$. If there was a collision, the electron is moved in the electric field to the collision time point $t_{\text {col }}$. Elastic, excitation and ionization collisions are considered. Electron is assumed to be scattered isotropically and in the excitation and ionization collision it also looses energy. After the collision the electron is continued to be traced, using the new velocity vector, until it reaches the end of $\Delta t_{\mathrm{elm}}$. In the ionization collision a new electron is created and it randomly shares the remaining energy with the parent electron. The angular distribution of it's emission is also taken to be isotropic. When the parent electron finally reaches the time $t+\Delta t_{\mathrm{smp}}$, all the electrons created during $\Delta t_{\mathrm{smp}}$ are traced in the above described manner from their time of creation until they all reach the time $t+\Delta t_{\mathrm{smp}}$.

The simulation ends when all the electrons are captured by the anode wire. 
Similar method was developed by Groh[4] from where we have taken the basic idea. In it's original form it is not however suitable for the gas pressures considered in this work. The time step in his work is based only on the mean free path and not on the variation of the electric field. This is adequate at 760 torr. At the gas pressures considered by us, the mean free path becomes so large that the electric field varies significantly on this distance especially in the vicinity of the anode wire and this has to be taken into account. Also his time step is calculated using only the average energy of all the electrons at a given time. We calculate this independently for each electron.

\section{Input data}

The simulations have been done for half inch TEPC counter with the cathode diameter of $1.27 \mathrm{~cm}$ and the anode diameter of $7 \mu \mathrm{m}$. The counter was assumed to be filled with methane. Methane was chosen because the electron-molecule cross-sections are relatively well known. The following cross-section measurements were used:

- elastic between $0.001 \mathrm{eV}$ and $1 \mathrm{eV}$ electron energy from Schmidt 91 [5], between $1.5 \mathrm{eV}$ and $100 \mathrm{eV}$ from Tanaka $91[6]$ and for energy range $100-500 \mathrm{eV}$ from Sakae 89 [7].

- ionization from Orient, 1987 [9]

- excitation from $[5,4,8]$

These cross-section data are summarized in Fig. 1

\section{Results}

\subsection{Study of the avalanche development}

Monte Carlo simulations of the avalanche development starting with one electron has been made under different operational conditions of the counter i.e different gas pressures and applied anode voltages. Particular attention has been paid to the dependence of the various features of the avalanche development on the gas pressure for a given voltage. The coordinate system of the counter has been chosen in such a way that the 
anode wire is perpendicular to the $x, y$ plane and goes through the origin. For a given operational conditions of the counter around 100 Monte Carlo runs were done starting with one single electron (primary electron) released at point $(4000 \mu m, 0,0)$ and time $t=0$. During each run the following quantities have been recorded:

- The complete trajectory of the primary electron,i.e. the $x, y, z$ coordinates for all time steps $\Delta t_{\mathrm{elm}}$.

- The coordinates at which the positive ions were created.

- Every few ps, the coordinates of the electrons existing at that time $t$ were sampled and recorded.

These recorded values allow a comprehensive analysis of the spatial and the temporal development of the avalanche, as will be discussed in the following paragraphs. The recorded quantities were plotted using the graphics package PAW [12] and inspected. From this we conclude the following:

\subsubsection{Spatial characteristics}

In Figures 2-7 the results of the M.C. calculations for different gas pressures and for voltages of $700 \mathrm{~V}$ and $450 \mathrm{~V}$ are shown. The following features can be observed:

- The extension of the avalanche along the anode wire (along the $z$ axis) increases with decreasing gas pressure, reaching the size of $2 \mathrm{~mm}$ for the lowest pressure (see Fig. 7). This expansion is linearly proportional to the inverse value of gas pressure.

- the avalanche is also expanding in the $x, y$ plane with decreasing gas pressure. However, this expansion is limited, for gas pressures down to 6.9torr, to a region with radius of about $150 \mu \mathrm{m}$ which, in the following, we will call proper avalanche region.

- the region from the first ionization down to the proper avalanche region is however expanding significantly. In the following, we will call this region the seeding region. In this region only a few electrons(seeding electrons) are created. These have however significant consequences for the further development of the avalanche as a whole (see below). 
- in all cases, the electron avalanche always completely surrounds the anode wire. This is very different situation from the conventional counters (operating usually at 760torr) where the avalanche is developing and contained on the side of the anode wire from which the primary electron is approaching. This effect is increasing with decreasing gas pressure which is caused by the fact that due to the increasing mean free path between the collisions with gas molecules, an electron has more chance to miss the wire and cause ionizations on the other side of the wire.

- the above effect gets very pronounced at low pressures, when it can happen that a single electron will circle the anode a few times before it is captured. This can be clearly seen on Fig. 6 .

\subsubsection{Time characteristics}

Figs. 8 and 9 show a typical time development of an electron avalanche for two different gas pressures. The horizontal axis is the time counted from the point of the release of the primary electron in psecs. On the vertical axis are for each time sample point plotted $x$ coordinates of all existing electrons at that time. Both figures have the same scale for the horizontal and the vertical axis. In particular, they span the last $6000 \mathrm{ps}$ of the avalanche. From these figures one can clearly distinguish 3 different stages or regions in the avalanche development:

drift region where the primary electron drifts towards the anode being affected only by the elastic and excitation collisions with the gas molecules.

proper avalanche region where 95 percent of the electron-ion pairs are created $(50 \mu \mathrm{m}$ for 69 torr, $150 \mu \mathrm{m}$ for 13.8 torr).

seeding region between the point of the first ionization of the primary electron and the proper avalanche region where only a few seeding electrons are created. Each of these seeding electrons gives subsequently rise to a partial avalanche in the proper avalanche region.

The time development of these partial avalanches can be more clearly seen from Figs. 10 and 11 which show the time distributions of ion creation. To complete the picture, Figs. 12 and 13 show the time developments in more details zooming on two 
partial avalanches in each case. In these figures also the points where the positive ions were created are shown.

Until now only two regions in avalanche development have been recognised i.e the drift and avalanche region and indeed at normal gas pressures where the proper avalanche and seeding regions merge together, this is sufficient. The seeding region becomes however very important at low pressures and provides an explanation for the behaviour of the various parameters describing the avalanche (see below).

\subsection{Statistical study of avalanche parameters}

As described above, around 100 runs were done for various operational conditions of the counter always releasing single electron from $4000 \mu \mathrm{m}$ on the $x$ axis. At the end of each run, when all the created electrons were captured by the anode, the recorded quantities were computer analysed and a number of parameters describing the avalanche were calculated. Here we will look at 3 of these, namely : gain and radial and time extent of the avalanche.

\subsubsection{Gain}

This is simply defined as the total number of electrons created in the avalanche. Figs. 14 and 15 show histograms of gain for two different gas pressures. As has been well known, the average gain is increasing with decreasing gas pressure in spite of the number of gas molecules decreasing. This has been explained in [1] by the fact that at low pressures many electrons turn around the anode wire thus having more chance to create further electrons. We find that this indeed is the case but this effect takes only place inside the partial avalanches. It results in partial avalanches having approximately same average number of ions in the considered gas pressure range. The real increase in the gain comes however from the increase of seeding region with the consequential increase in the number of partial avalanches. The attempt to make the statistical description of electron avalanche gain were done by Byrne who introduced the parameter $b$. The curves on Figs. 14 and 15 is the distribution function as predicted by [11] namely:

$$
f(\text { gain }) \sim \operatorname{gain}^{\frac{1}{b}-1} \exp \left(\frac{\operatorname{gain}}{b<\operatorname{gain}>}\right)
$$

where $\langle$ gain $>$ is the average gain and $b$ is fitted parameter. As can be seen, the agreement between the data and the theoretical curves is very good. 


\subsubsection{Radial extent of avalanche $\Delta R$}

This we defined as a cylindrical region of such a radius from the anode within which 95 percent of electron-ion pairs are created (proper avalanche region). Figs. 16 and 17 show histograms of $\Delta R$ for two different gas pressures. As expected, the average value of $\Delta R$ is increasing with decreasing gas pressure but not so fast. An interesting feature of both distributions is how well the proper avalanche region is defined, i.e. how narrow both distributions are.

\subsubsection{Time extent of avalanche $\Delta T$}

We defined this as the time interval within which 95 percent electron-ion pairs are created. The end of the time interval is chosen to be the time when all the electrons were captured by the anode. The Figs. 18 and 19 show histograms of $\Delta T$ for two different gas pressures. The fact that the average $\Delta T$ is increasing with decreasing gas pressure is due to two effects:

- The time extent of each partial avalanche is increasing because the electrons are circling the anode and it takes consequently more time before they are captured.

- Increase of the seeding region has the consequence that a number of seeding electrons are produced far from the anode. They then drift towards the proper avalanche region reaching it at different times and the $\Delta T$ becomes dominated by the fluctuations in the drift time.

This later effect also explains the large fluctuations in $\Delta T$ at low pressures.

\subsection{Dependence of the average values of $\overline{\text { Gain }}, \overline{\Delta R}$ and $\overline{\Delta T}$ on the release point $r_{\text {init }}$ of the primary electron}

Study of this dependence is clearly important in order to understand the performance of a counter since the primary ionizing track can occur in any part of it's volume. We have considered 3 operational conditions of the counter namely 69torr , 34.5torr and 13.8torr, all of these at the anode voltage of $700 \mathrm{~V}$. This time we have however also considered different values of the distance of the release point of the primary electron from the anode $r_{\text {init }}$. Similarly as before, around 100 runs were done for a given pressure 
and $r_{\text {init }}$. For each of the runs the values of Gain, $\Delta R$ and $\Delta T$ were calculated and from these for each set of 100 runs the average values of $\overline{G a i n}, \overline{\Delta R}$ and $\overline{\Delta T}$.

\subsubsection{Gain}

The results are shown in Fig. 20. Even though we suffer from the lack of statistics, the characteristic dependence on $r_{\text {init }}$ is clearly visible,namely the plateau region followed by a drop towards the low values of $r_{\text {init }}$ for the two lower pressures. This is caused by the fact that the seeding region is being reduced with the consequential reduction in the number of partial avalanches. The effect is obviously stronger at low pressures. On Fig. 20 also the crossover point bellow $1000 \mu \mathrm{m}$ is noticeable. The fact that the crossover point occurs above $500 \mu \mathrm{m}$, which is still well above the proper avalanche region even for 13.8torr (see Fig. 17) , makes us believe that the average number of ions in a partial avalanche is actually decreasing with decreasing gas pressure and as was already pointed above, the dependence of the $\overline{\text { Gain }}$ on pressure is dominated by the size of the seeding region.

\subsection{2 $\overline{\Delta R}$}

It comes as no surprise, that the average size of the proper avalanche region is more or less independent of $r_{\text {init }}$ for all pressures as can be seen from Fig. 21.

\subsection{3 $\overline{\Delta T}$}

The behaviour of $\overline{\Delta T}$ is remarkably similar to that of $\overline{\text { Gain }}$ as can be seen from Fig. 22 . Drop towards the small $r_{\text {init }}$ for low pressures can again be explained by the expansion of the seeding region at low pressures. Releasing the primary electron sufficiently far from this region, it can take the advantage of it's full extent and will produce on average the same number of seeding electrons resulting in $\overline{\Delta T}$ being independent of $r_{\text {init }}$. By releasing the primary electron within this region, the number of seeding electrons drops and since the avalanche time is dominated by the fluctuations in their drift velocity it has the consequence that the $\overline{\Delta T}$ drops also. There is a hint of a cross over point but this will have to be investigated further. 


\section{Conclusions}

We have presented results from a study of various electron avalanche parameters in a microdosimetric counter. The technique is based on the Monte Carlo simulation of the motion of electrons in electric field and their interactions with the molecules of gas. It allows us to study all space and temporal aspects of electron avalanche development since all the electrons are traced simultaneously in time and their coordinates and velocities are recorded. This approach depends only on the measured electron-molecule cross-sections. These results are already pointing to several important characteristics of the electron avalanche behaviour, in particular the existence of the seeding region in the avalanche where a relatively small number of secondary electrons are created. Each of these electrons gives subsequently rise to a partial avalanche in the proper avalanche region. This allows us to explain the dependence of gain and the time extent of electron avalanche on the gas pressure and the distance from the anode where the primary electron was released. The importance of partial avalanches has been already noted by Groh at al[3] but due to the lack of computer time they could not study this effect adequately. Also in their case the partial avalanches do not play such a significant role as at the gas pressures considered in this work.

The operational limits of TEPC counters at low gas pressures are due to the fact that with decreasing pressure the size of the seeding region is increasing and consequently the counter is becoming less and less proportional. Another negative effect contributing to the loss of proportionality is the avalanche spreading along the anode wire. The present technique should allow us to establish these operational limits and to study possible improvements in the construction of these counters.

At this point it is worth mentioning that the analysis is quite CPU time consuming. One run takes approximately 15 mins on VAX station 4000/90. It means that 1 point shown on Figs. 20,21 and 22 takes about 1day of CPU time.

We believe that this is the correct approach to fully understand the operation of these counters and we would like to investigate several aspects in full details in the near future such as the precise definition of a partial avalanche or the physical significance of the parameter $\mathrm{b}$ in the Byrne distribution. It should be noted that the method can be easily extended to other geometries other then cylindrical. This is simply done by providing the appropriate electric field map. 


\section{References}

[1] P. Ségur, I. Pérès, J. P. Boeuf and M. C. Bordage, Microscopic Calculation of the Gas Gain in Cylindrical Proportional Counters, Radiation Protection Dosimetry 29(1-2) (1989) 23-30.

[2] M. Matoba, T. Hirose, T. Sakae, H. Kametani, H.Ijiri and T. Shintake, Three Dimensional Monte Carlo Simulation of the Electron Avalanche around an Anode Wire of a Proportional Counter, IEEE Transactions on Nuclear Science Vol. NS32 No.1 (1985) 541-544

[3] J. Groh, E. Schenuit and H. Spitzer, Computer Simulation of the Electron Avalanche in Cylindrically Symmetric Electric Fields, Nuclear Instruments and Methods A 283 (1989) 730-734.

[4] J. Groh, Computersimulation der Elektronenlawine in zylindersymmetrischen elektrischen Feldern, Interner Bericht DESY FH1T-89-03 Mai 1989.

[5] B. Schmidt, J.Phys.B: Mol. Opt. Phys. 24 (1991) 4809-4820.

[6] H. Tanaka and L. Boesten, J.Phys.B: Mol. Opt. Phys. 24 (1991) 821-832.

[7] T. Sakae, S. Sumiyoshi, E. Murakami, Y. Matsumoto, K. Ishibashi and A. Katase, J.Phys.B: Mol. Opt. Phys. 22 (1989) 1385-1394.

[8] L. Vuskovic and S. Trajmar, Electron Impact Excitation of Methane, J.Chem.Phys. 78(8) (1983) 4947-4951.

[9] O. J. Orient, S. K. Srivastava, J.Phys.B: At.Mol.Phys. 20 (1987) 3923-3936.

[10] B. Schmidt, Ein Beitrag zur Erforschung des Elektrontansports in Gasen, Habilitationsschrift (Ruprecht-Karls Universitat Heidelberg, 1991).

[11] J. Byrne, Statistics of Electron Avalanches in the Proportional Counter, Nuclear Instruments and Methods 74 (1969) 291-296.

[12] PAW - Physics Analysis Workstation, CERN Program Library 


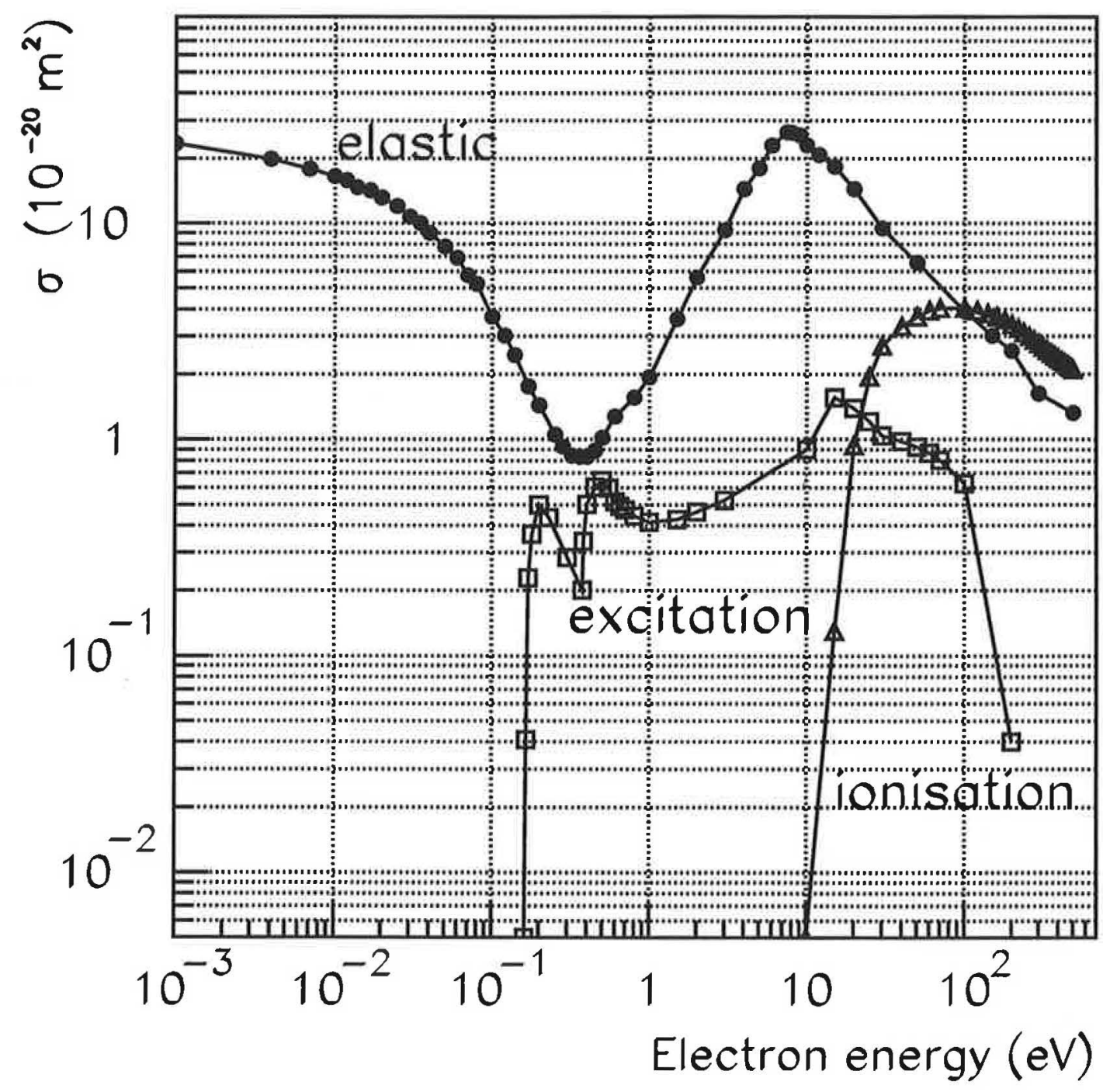

Figure 1: Electron molecule cross-sections in methane 


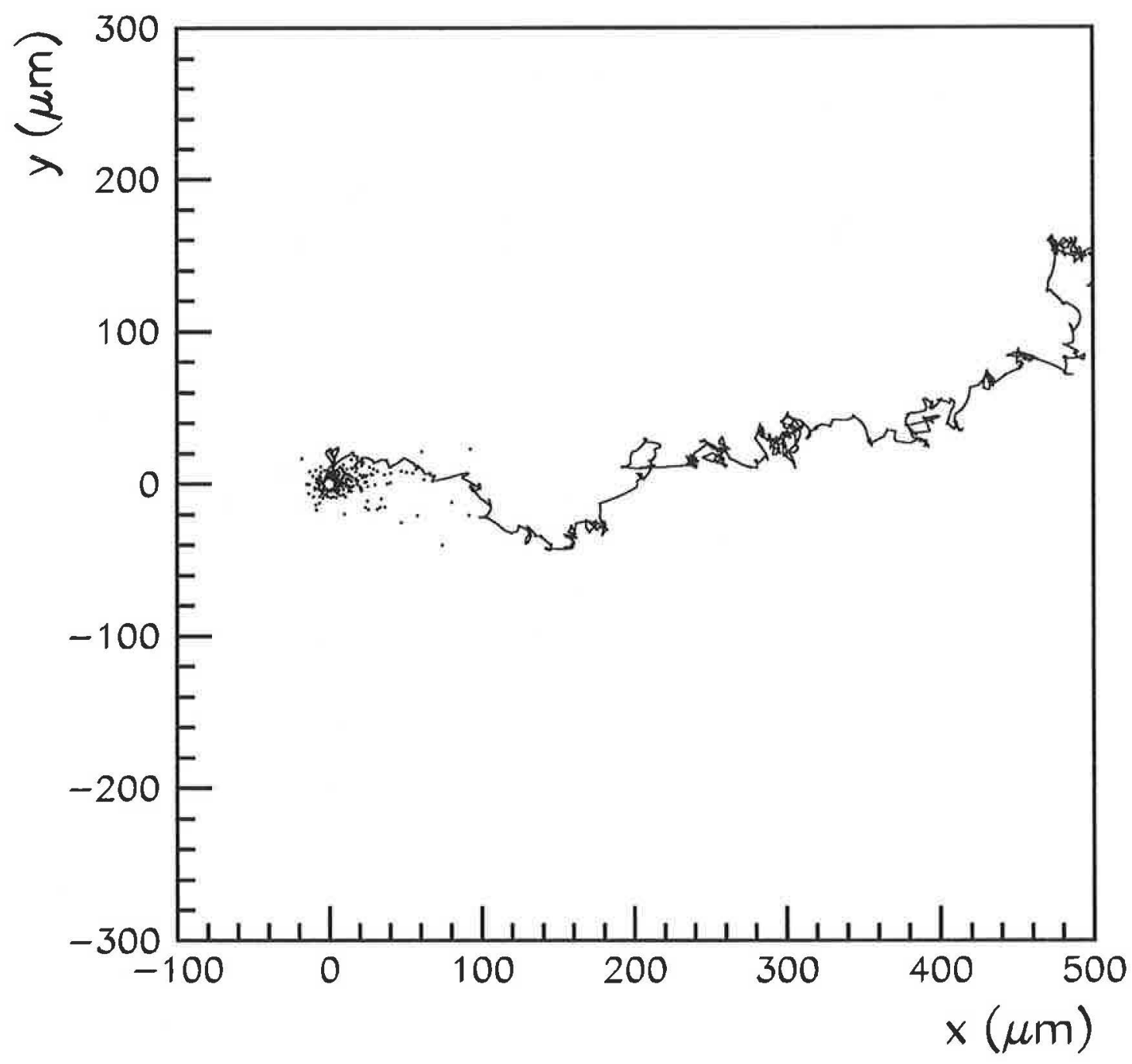

Figure 2: The path of the primary electron projected on the $x y$ plane. The points indicate the positions at which electron-ion pairs were created by secondary electrons. Calculated for 69 torr and $700 \mathrm{~V}$. 


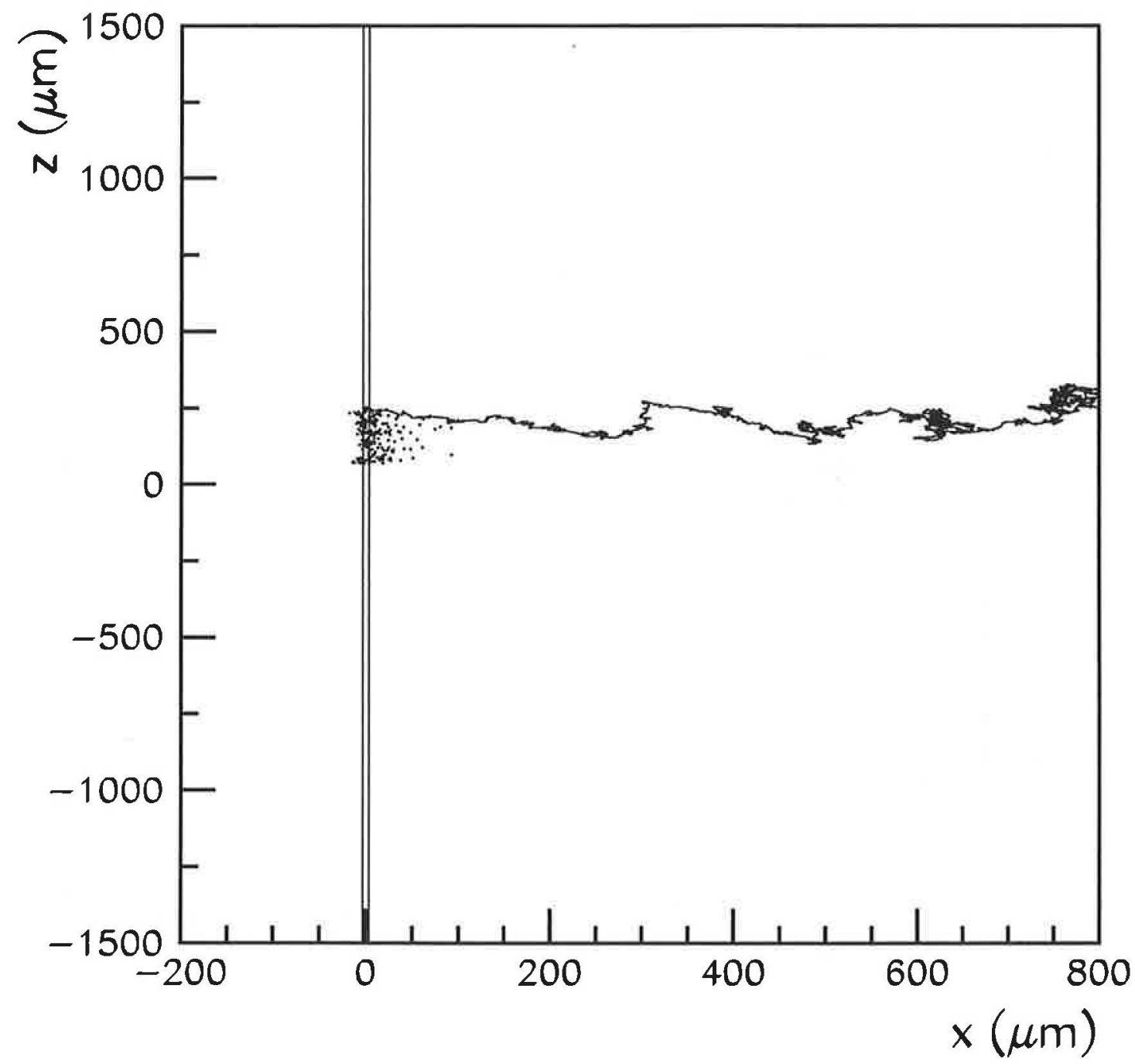

Figure 3: The path of the primary electron projected on the $x z$ plane. The points indicate the positions at which electron-ion pairs were created by secondary electrons. Calculated for 69 torr and $700 \mathrm{~V}$. 


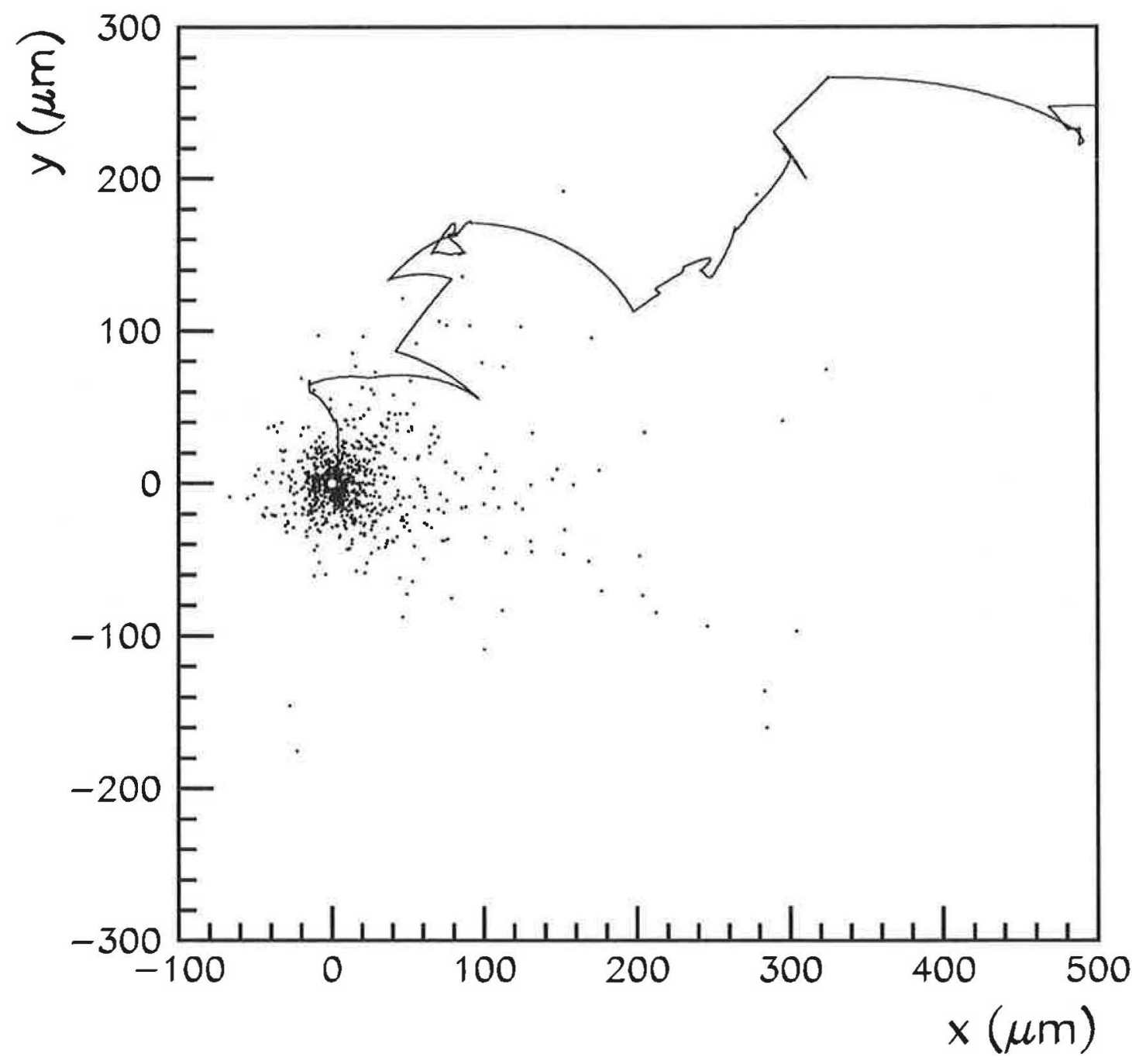

Figure 4: The path of the primary electron projected on the $x y$ plane. The points indicate the positions at which electron-ion pairs were created by secondary electrons. Calculated for 13.8 torr and 700V. 


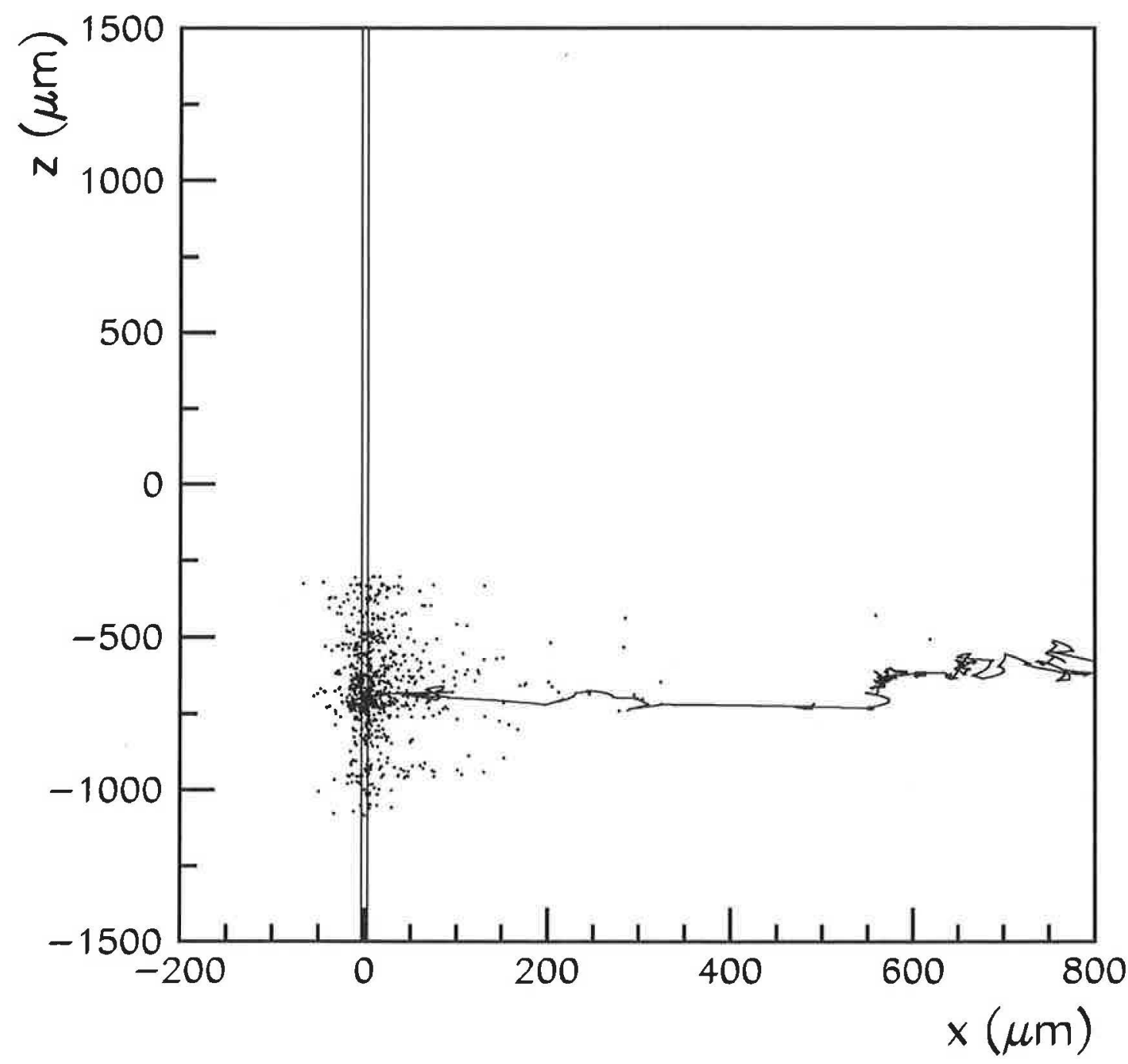

Figure 5: The path of the primary electron projected on the $x z$ plane. The points indicate the positions at which electron-ion pairs were created by secondary electrons. Calculated for 13.8 torr and 700V. 


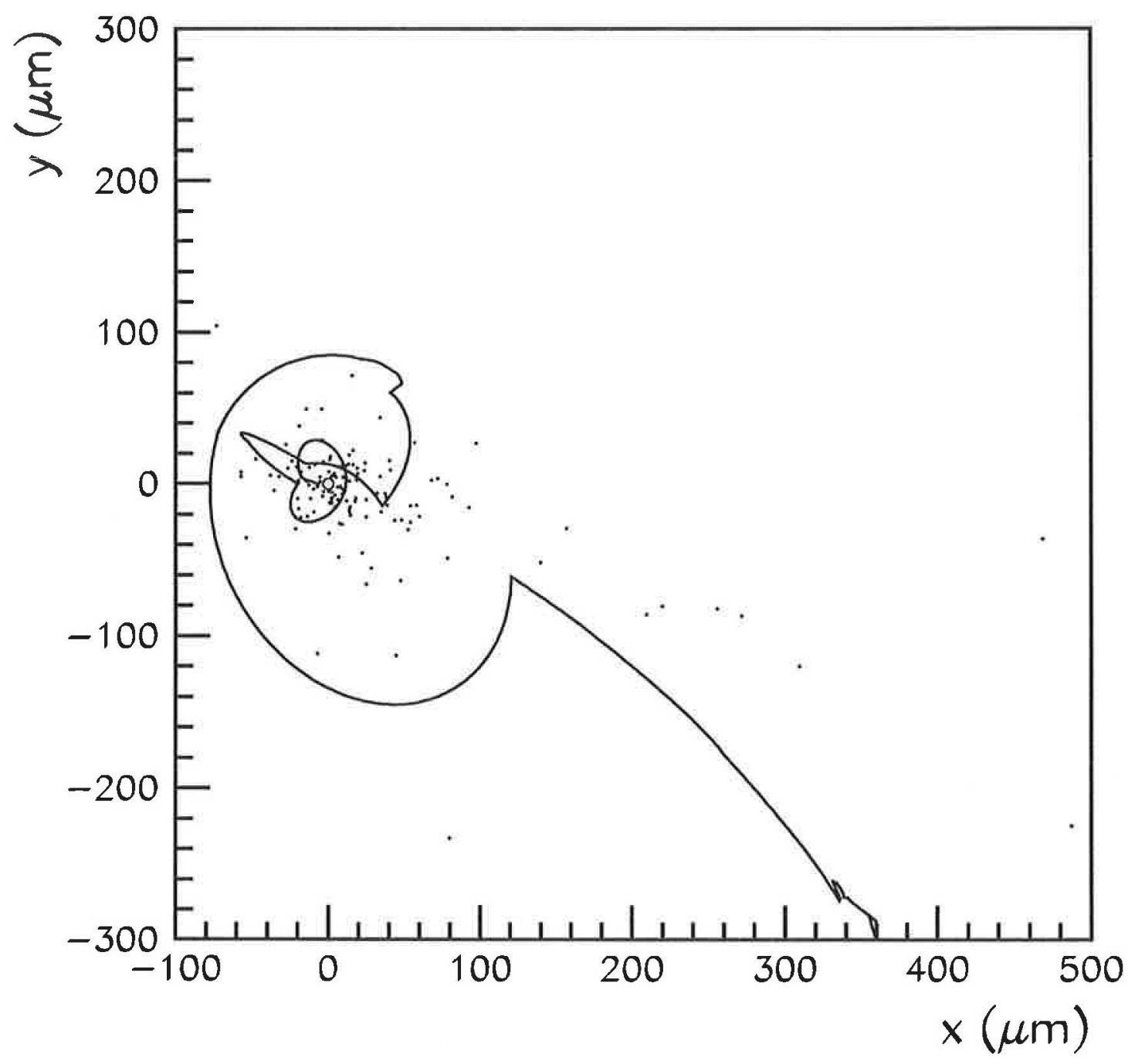

Figure 6: The path of the primary electron projected on the $x y$ plane. The points indicate the positions at which electron-ion pairs were created by secondary electrons. Calculated for 6.9 torr and $450 \mathrm{~V}$. 


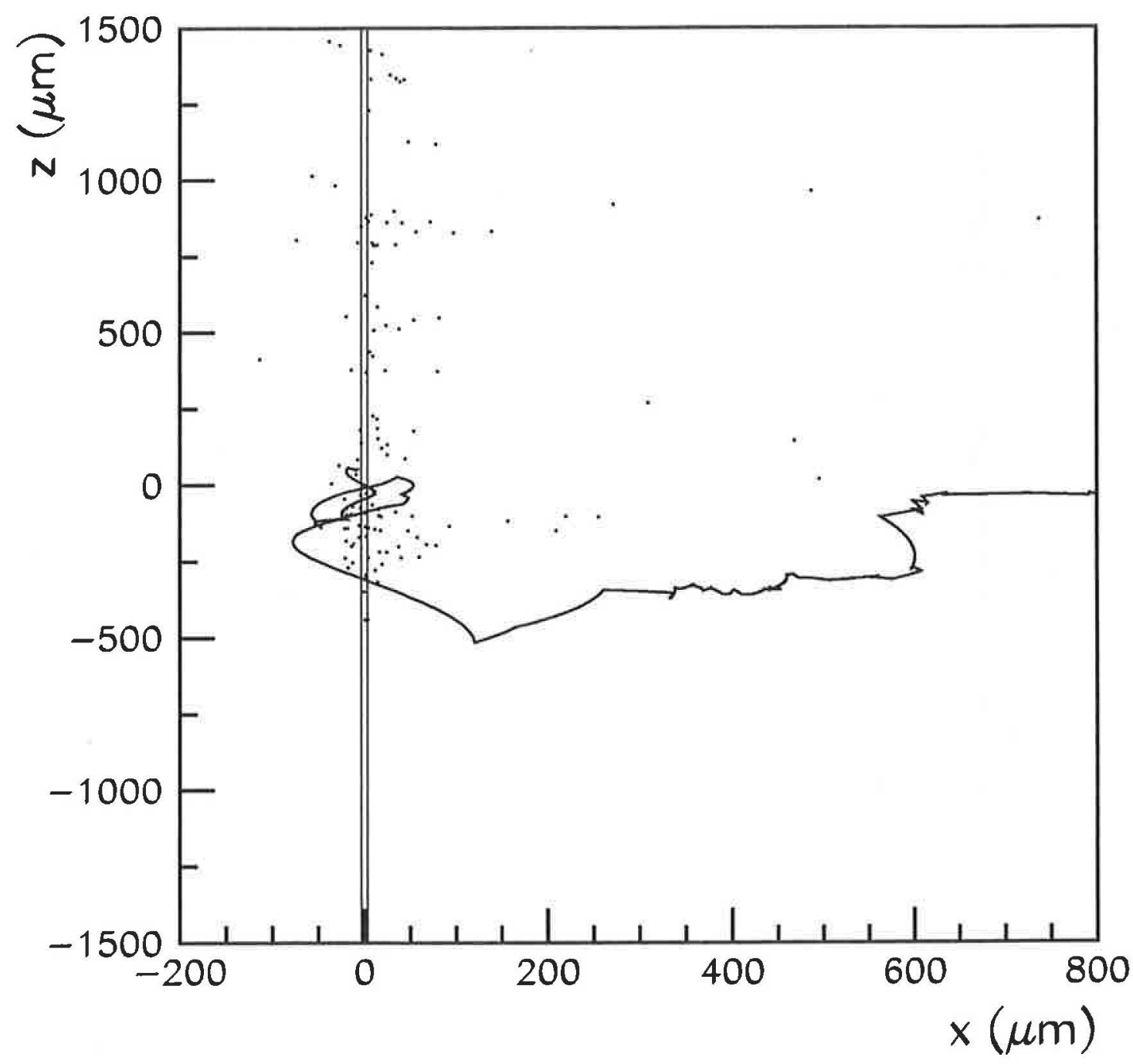

Figure 7: The path of the primary electron projected on the $x z$ plane. The points indicate the positions at which electron-ion pairs were created by secondary electrons. Calculated for 6.9 torr and $450 \mathrm{~V}$. 


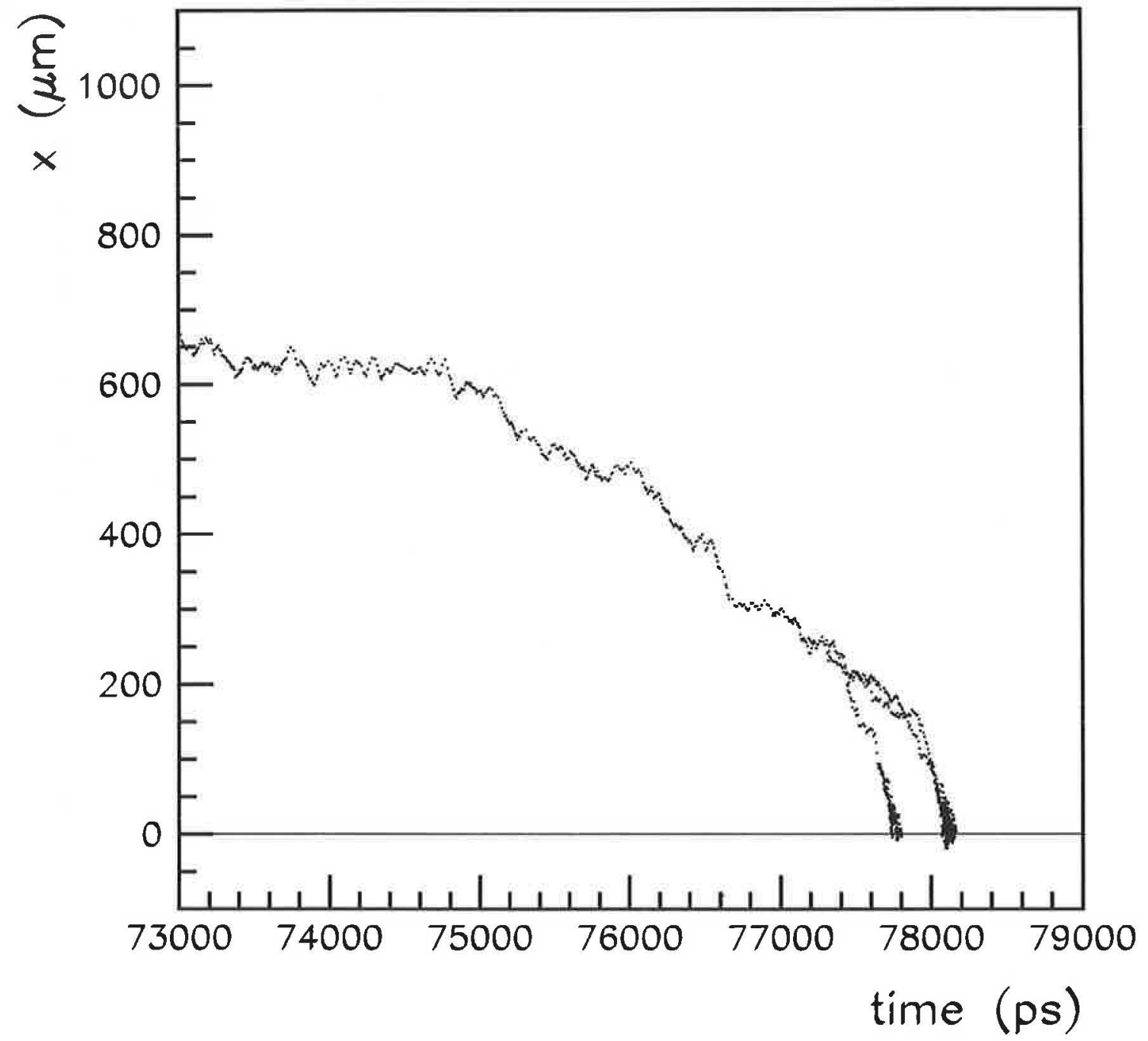

Figure 8: The space-time picture of avalanche development. The points are $x$ coordinates of all existing electrons at a given time. Calculated for 69 torr and $700 \mathrm{~V}$. 


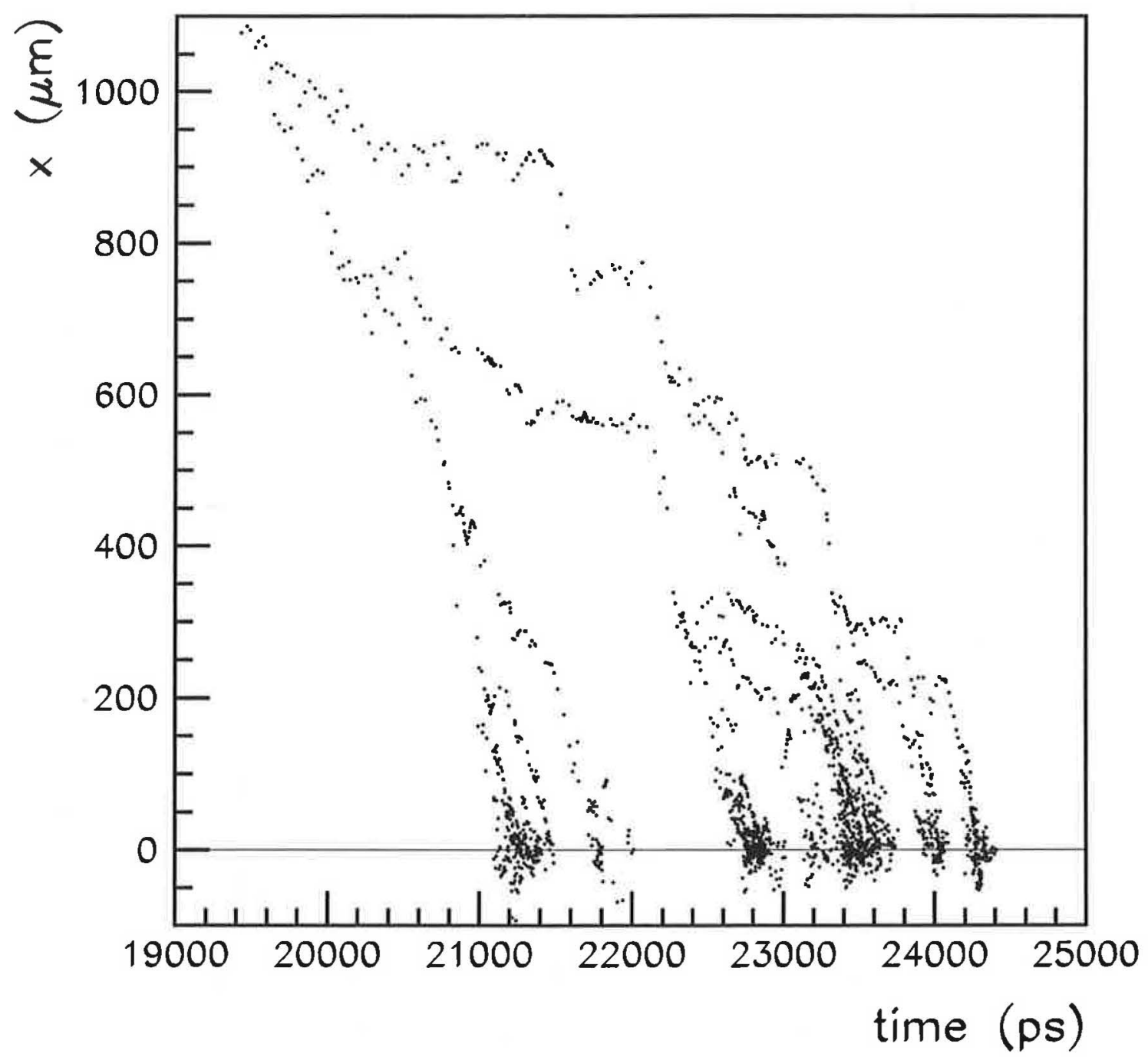

Figure 9: The space-time picture of avalanche development. The points are $x$ coordinates of all existing electrons at a given time. Calculated for 13.8 torr and $700 \mathrm{~V}$. 


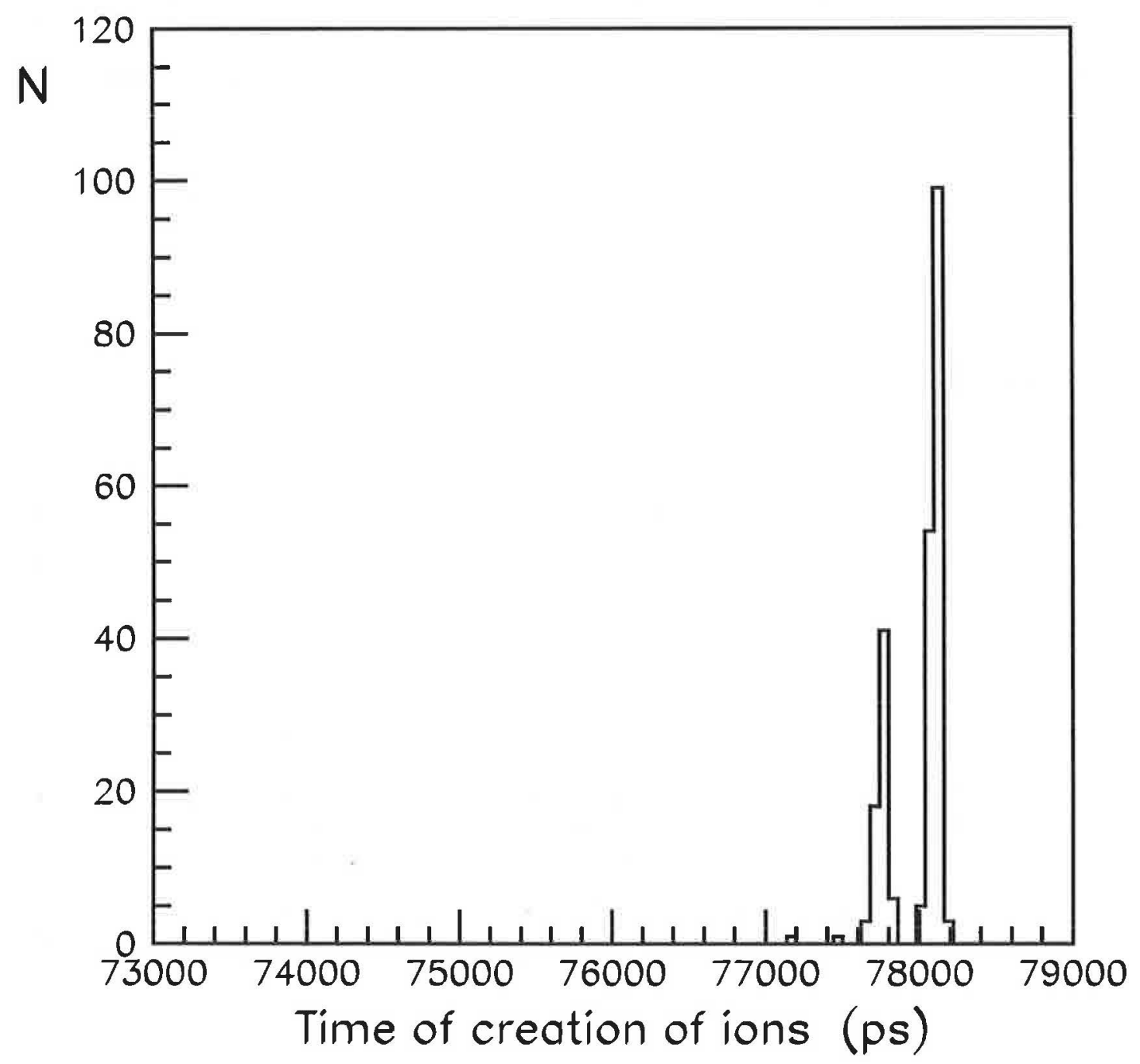

Figure 10: The time distribution of ion creation. Calculated for 69 torr and $700 \mathrm{~V}$. 


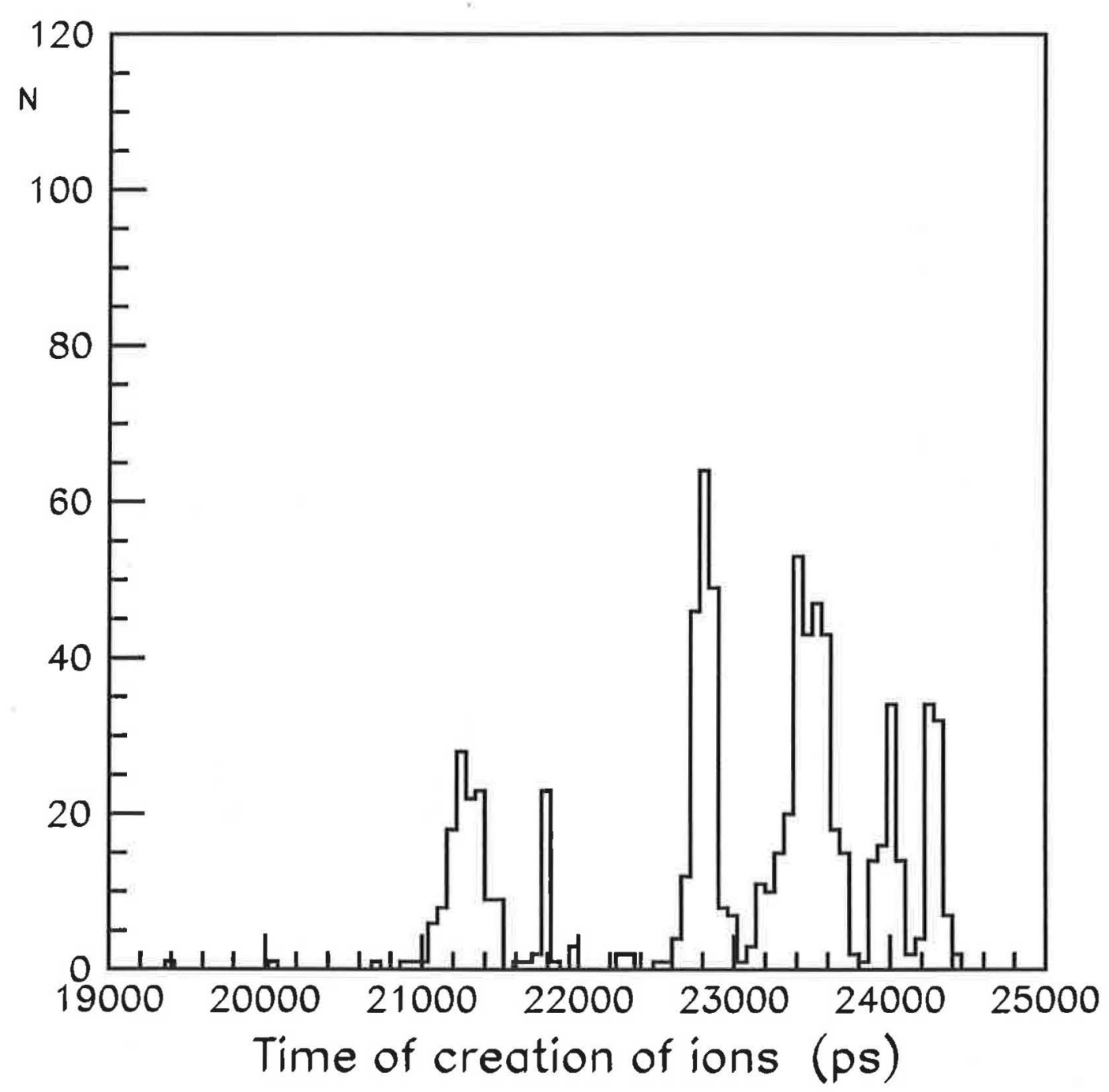

Figure 11: The time distribution of ion creation. Calculated for 13.8 torr and $700 \mathrm{~V}$. 


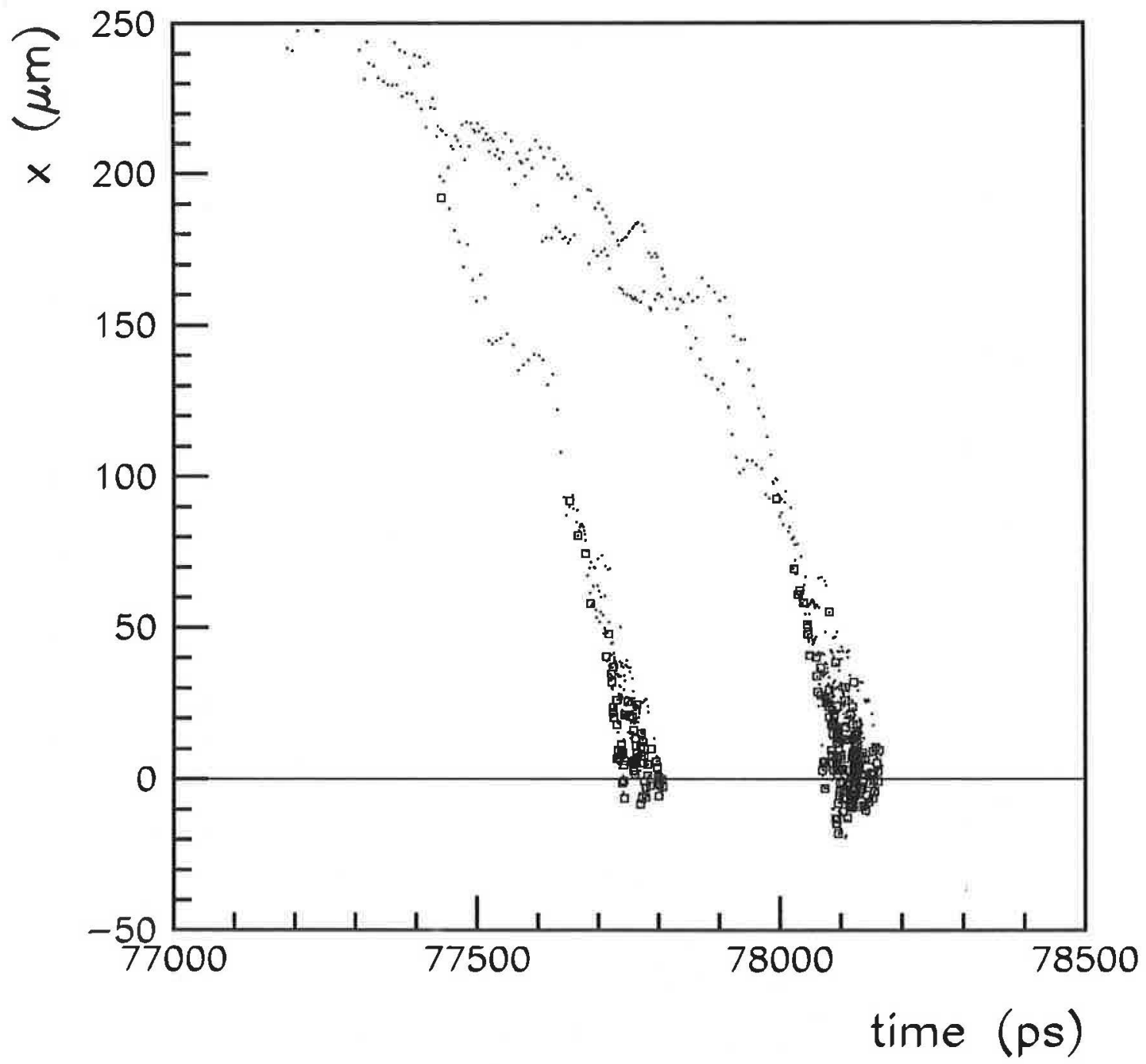

Figure 12: Same as fig. 8 but on a larger scale. The small squares indicate $x, t$ positions where ions were created. 


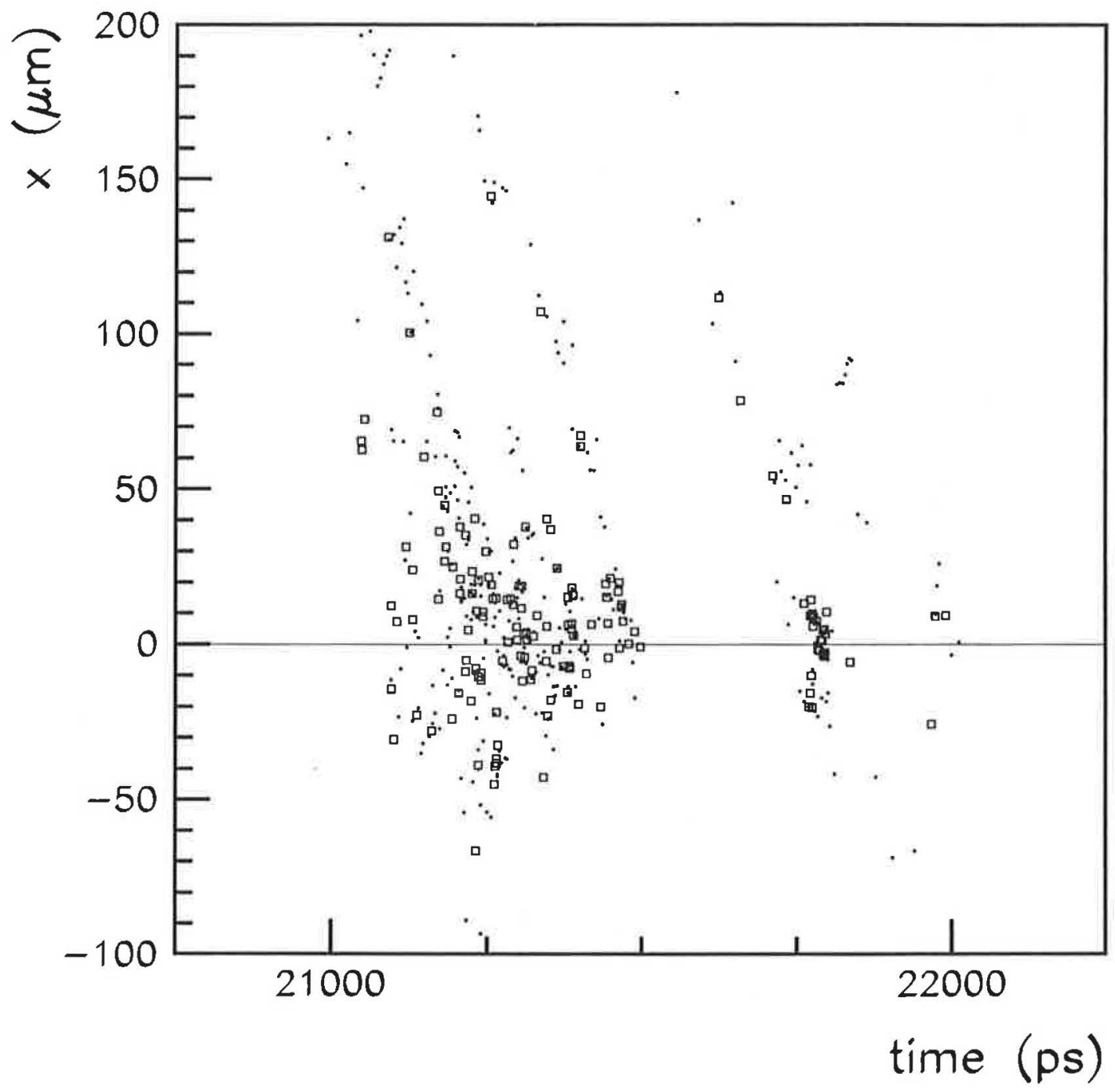

Figure 13: Same as fig. 9 but on a larger scale. Only the first two partial avalanches can be seen. The small squares indicate $x, t$ positions where ions were created. 


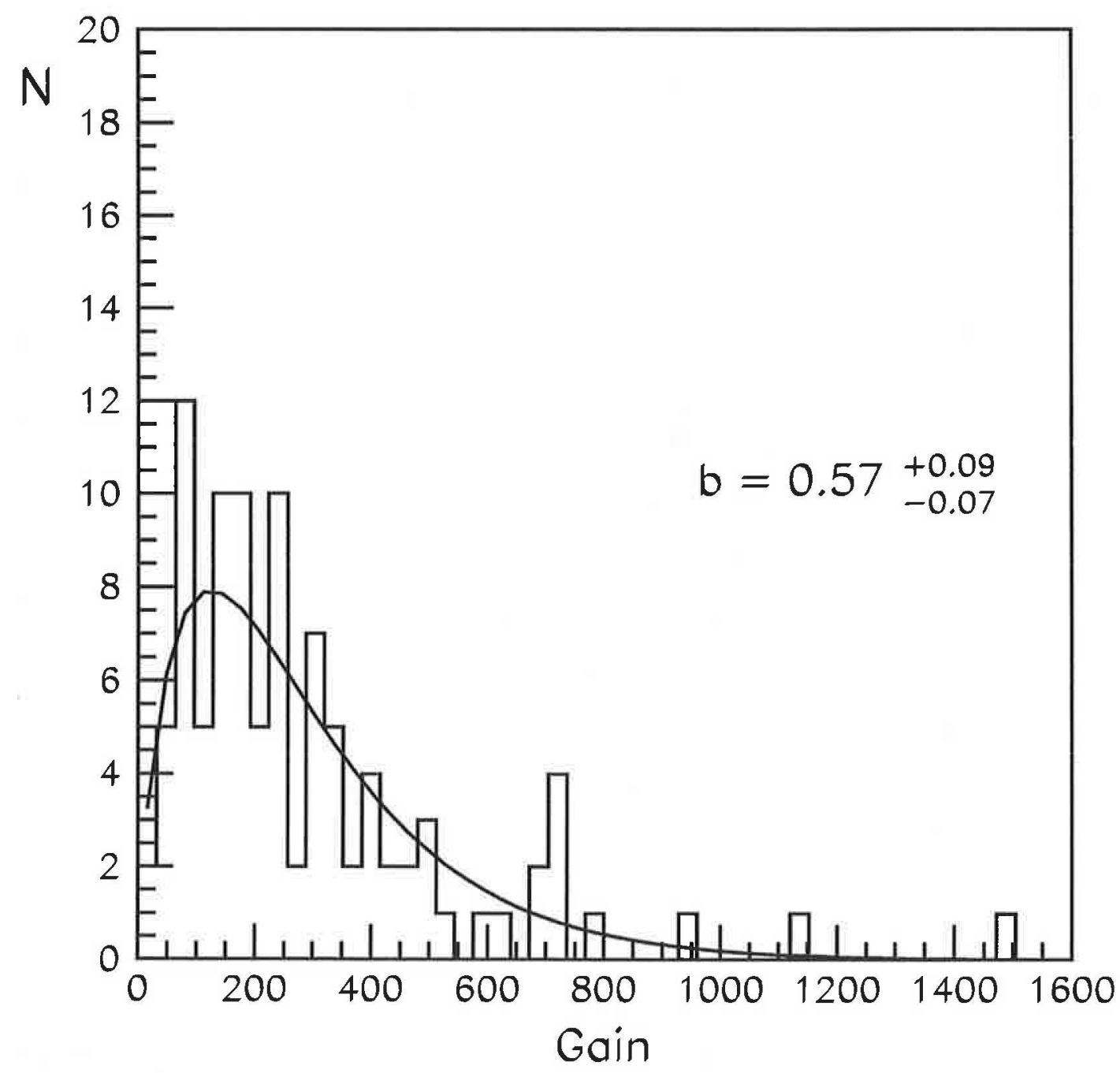

Figure 14: Histogram of Gain from single electron released at $4000 \mu \mathrm{m}$ from the anode at 69 torr and $700 \mathrm{~V}$. The curve shown is the Byrne distribution 


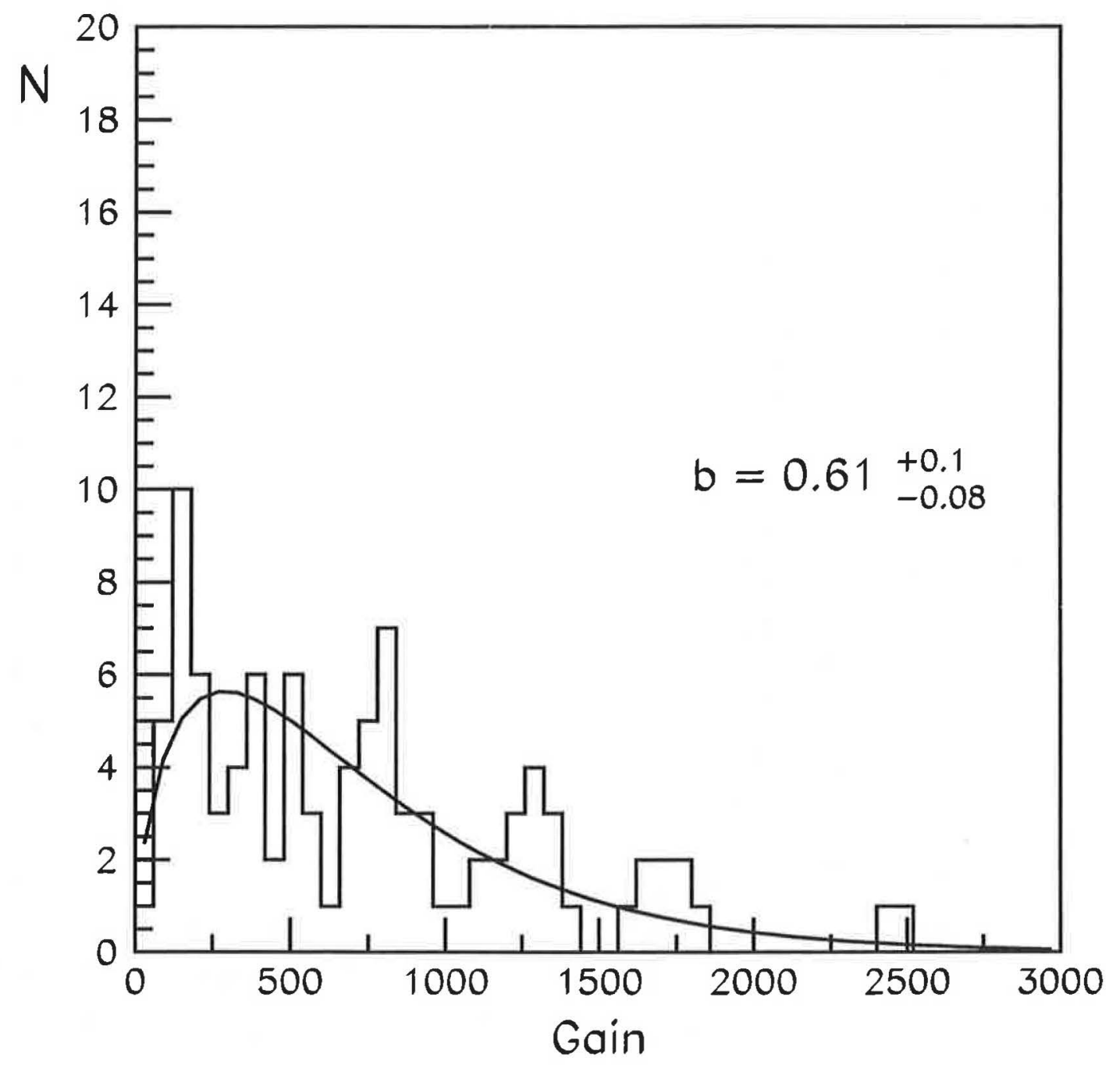

Figure 15: Histogram of Gain from single electron released at $4000 \mu m$ from the anode at 13.8 torr and $700 \mathrm{~V}$. The curve shown is the Byrne distribution 


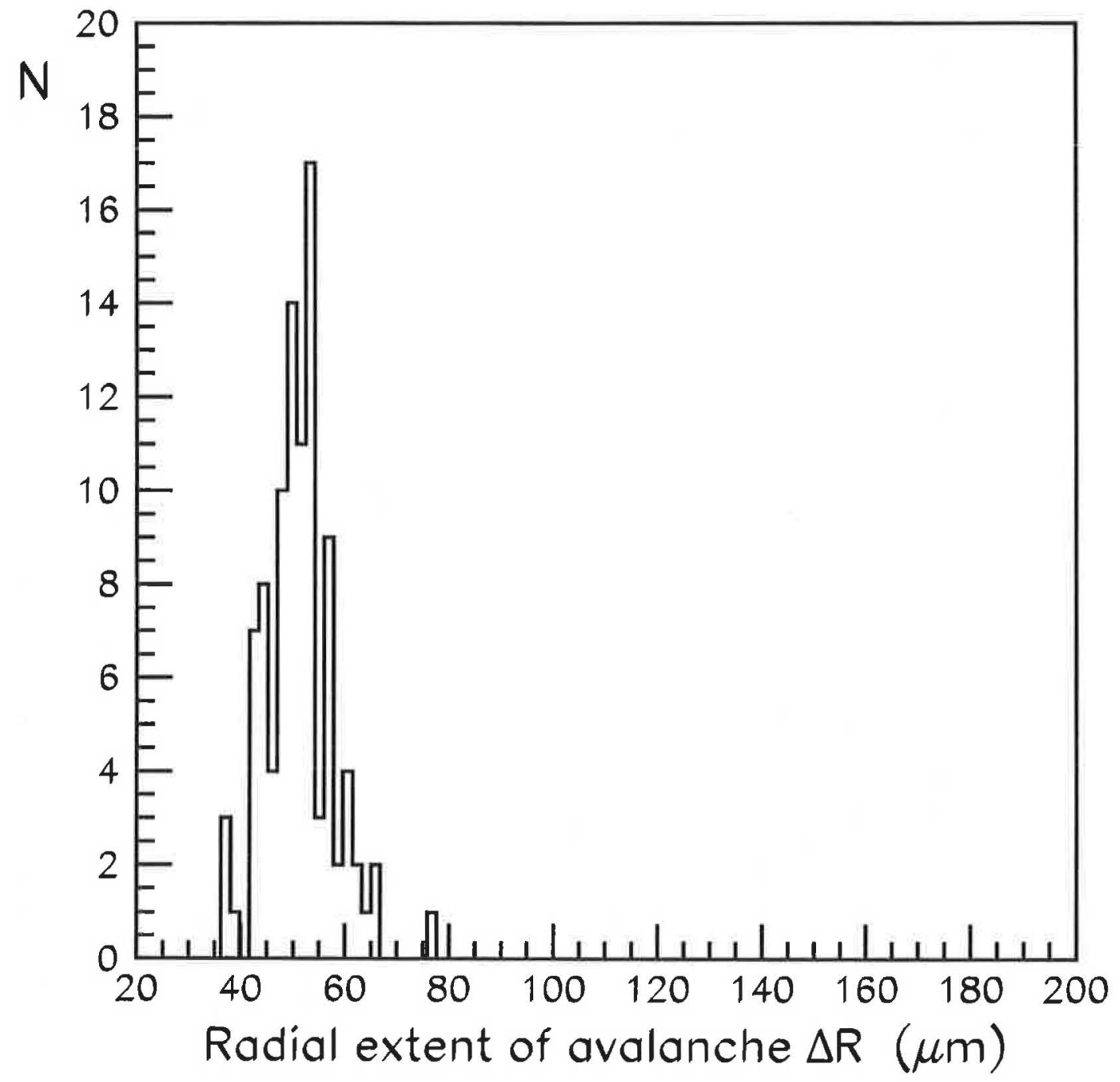

Figure 16: Histogram of the radial extent of avalanches from single electron released at $4000 \mu \mathrm{m}$ from the anode at 69 torr and $700 \mathrm{~V}$. 


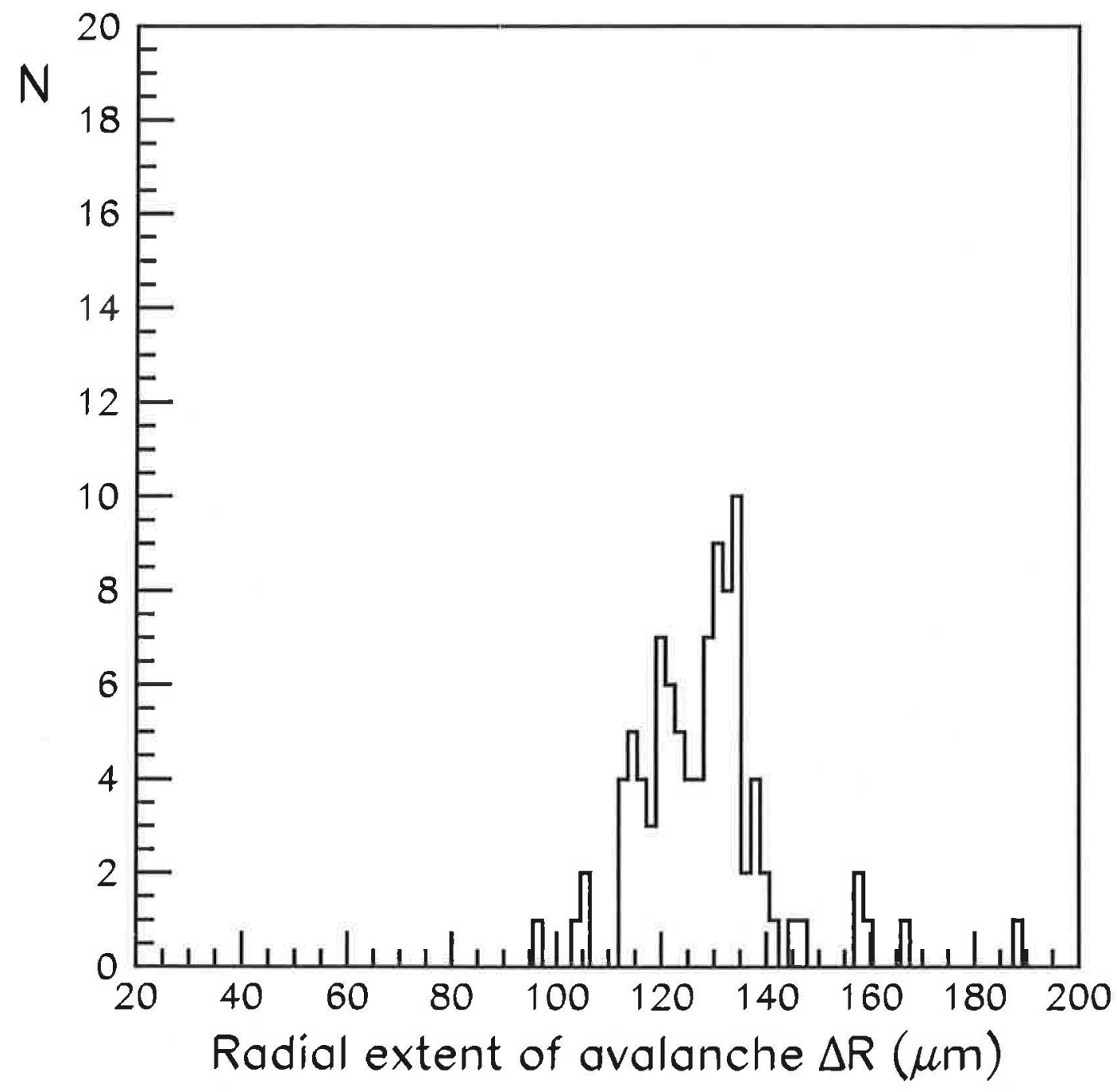

Figure 17: Histogram of the radial extent of avalanches from single electron released at $4000 \mu \mathrm{m}$ from the anode at 13.8 torr and $700 \mathrm{~V}$. 


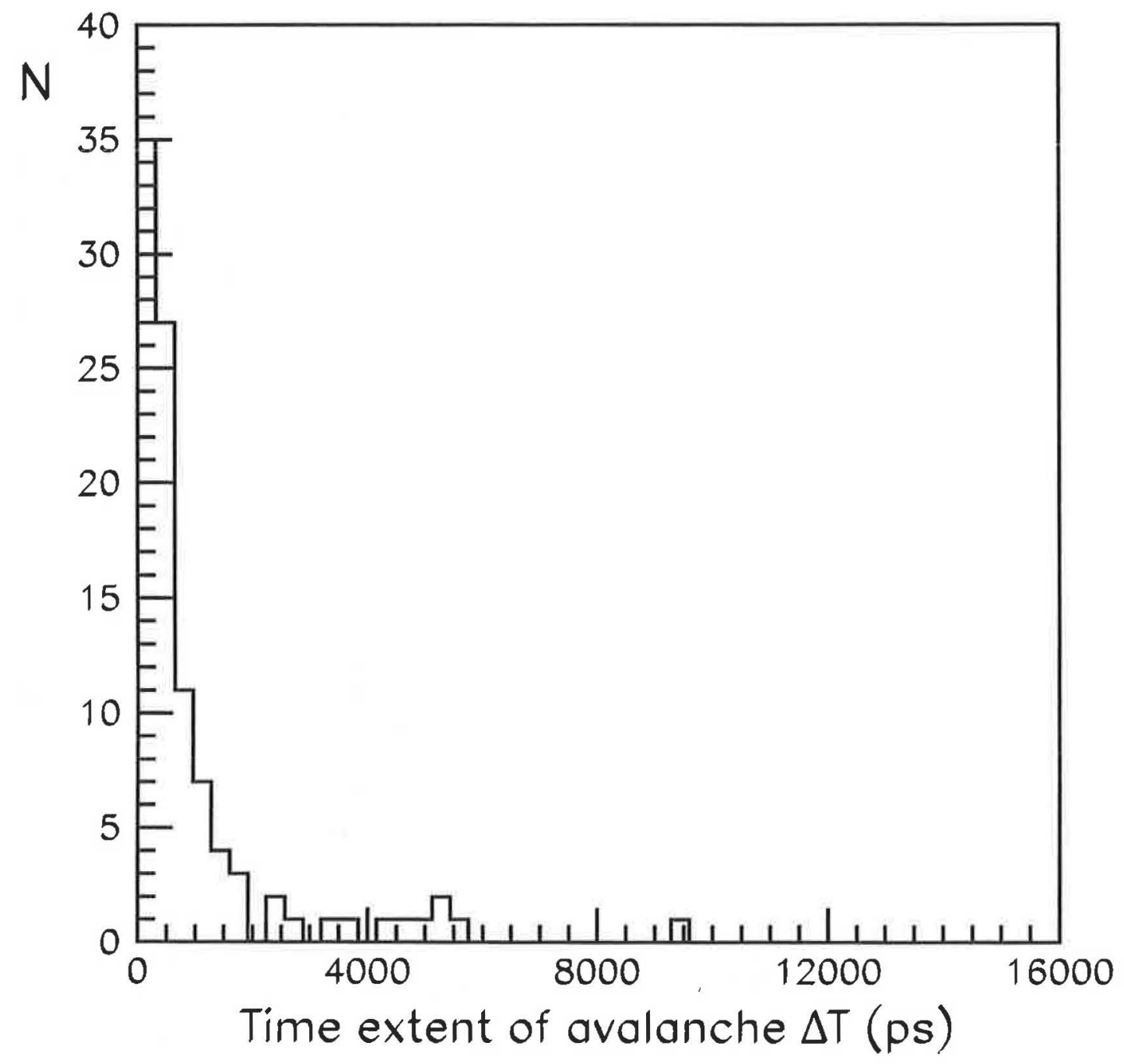

Figure 18: Histogram of the time extent of avalanches from single electron released at $4000 \mu \mathrm{m}$ from the anode at 69 torr and $700 \mathrm{~V}$. 


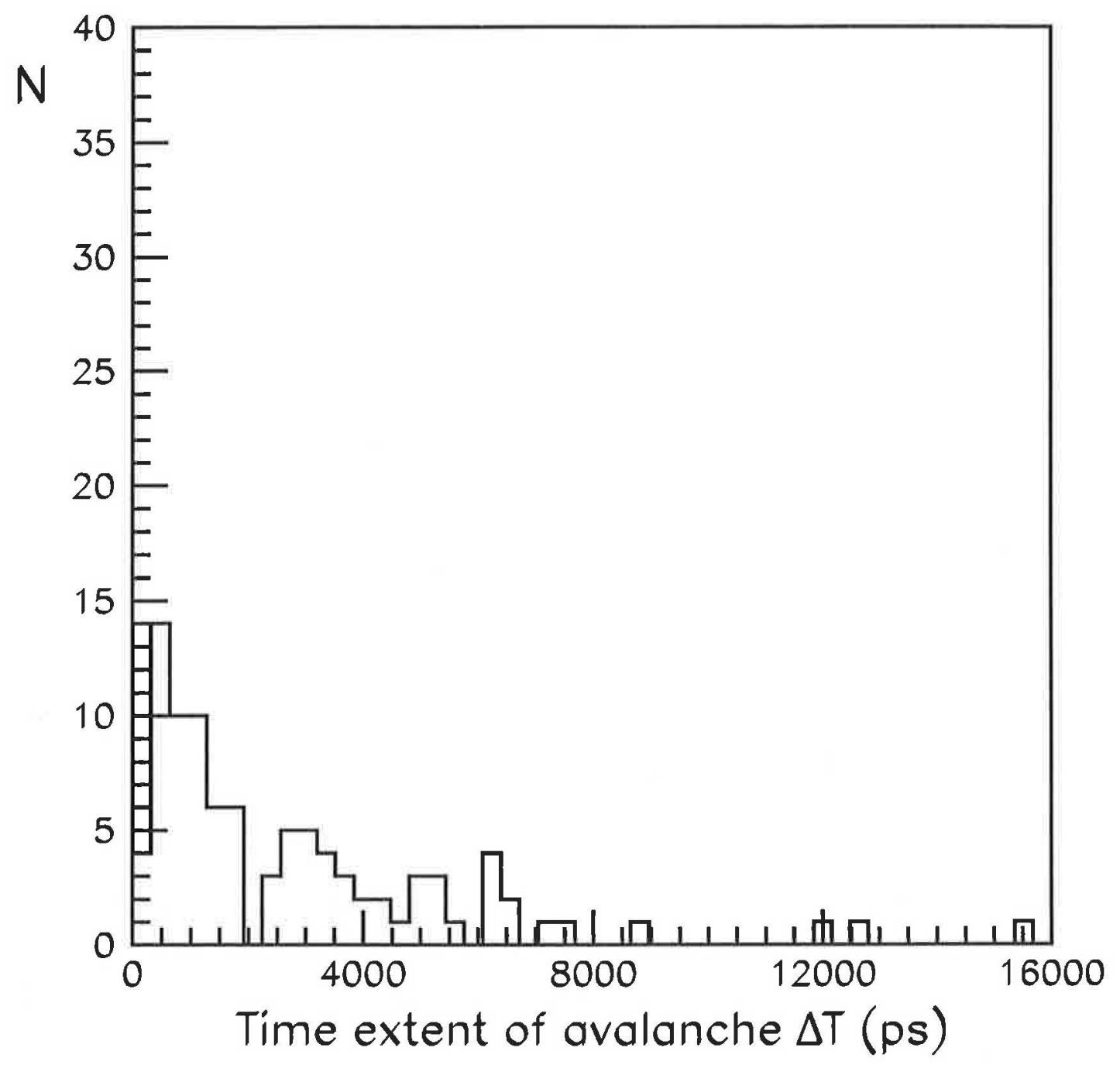

Figure 19: Histogram of the time extent of avalanches from single electron released at $4000 \mu \mathrm{m}$ from the anode at 13.8 torr and $700 \mathrm{~V}$. 


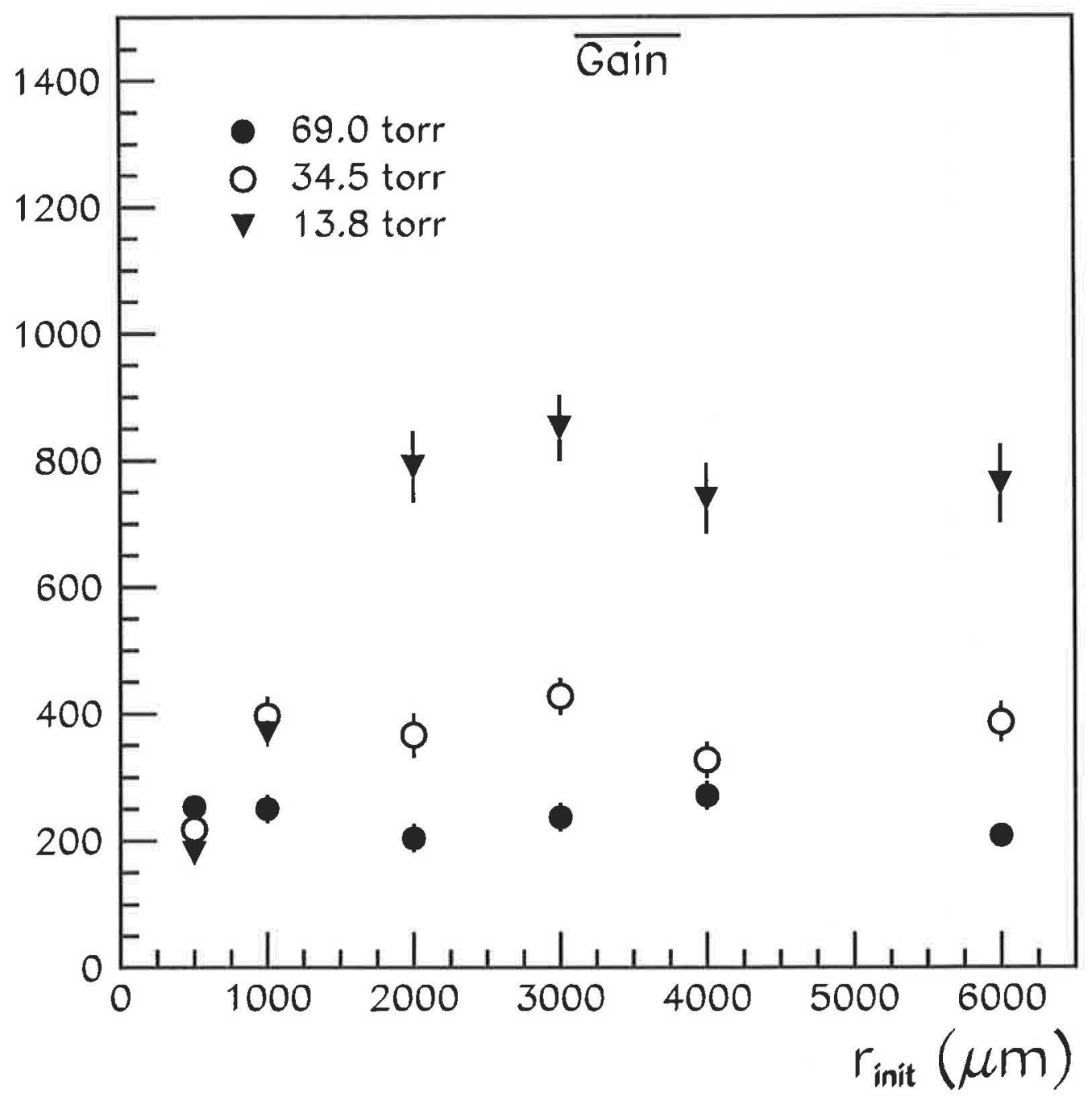

Figure 20: Dependence of the average gain $\overline{\text { Gain }}$ on the distance $r_{\text {init }}$ from the anode at which the primary electron was released. Calculated for 3 different gas pressures and anode voltage $700 \mathrm{~V}$. 


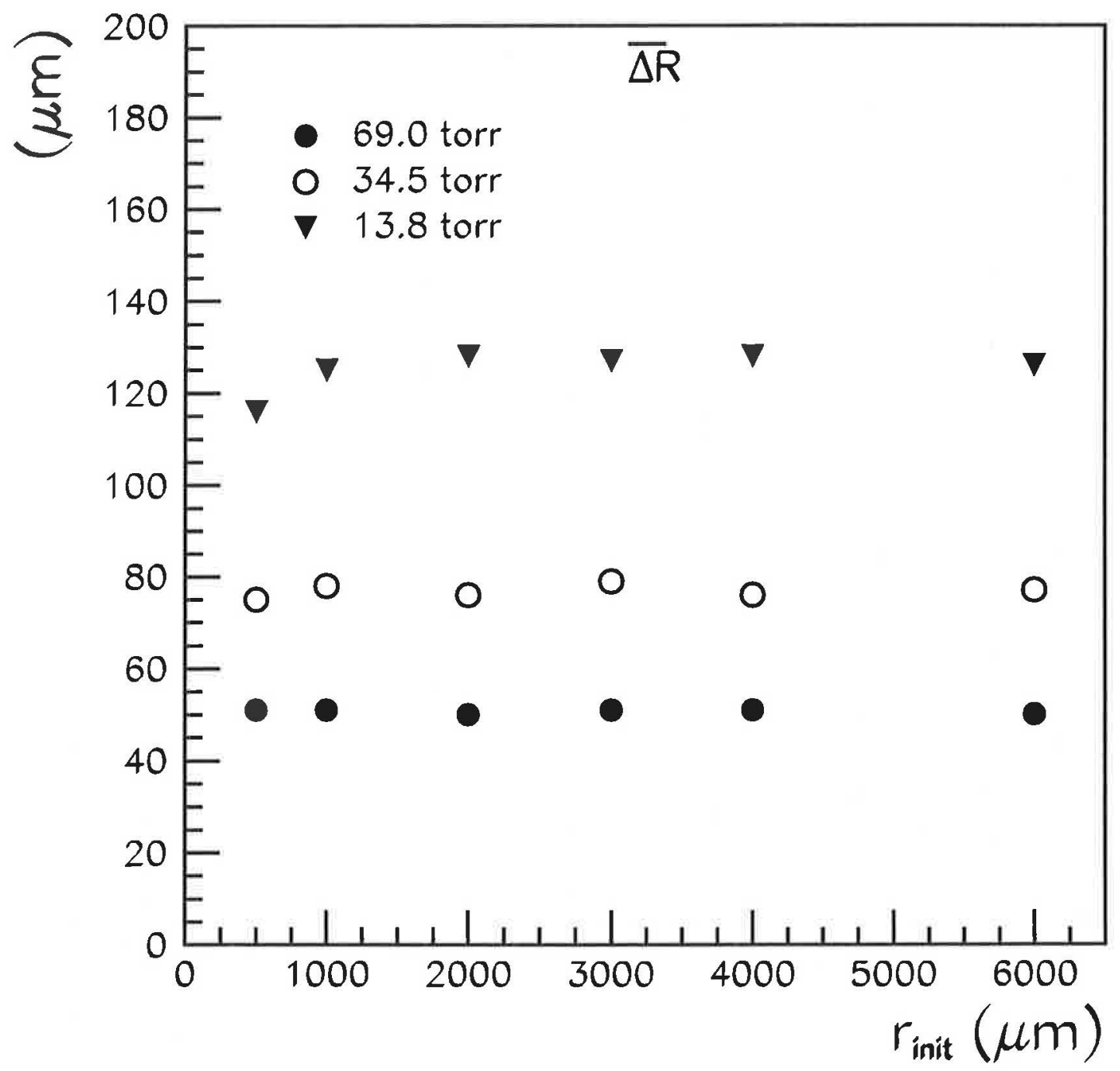

Figure 21: Dependence of the average radial extent of avalanche $\overline{\Delta R}$ on the distance $r_{\text {init }}$ from the anode at which the primary electron was released. Calculated for 3 different gas pressures and anode voltage $700 \mathrm{~V}$. 


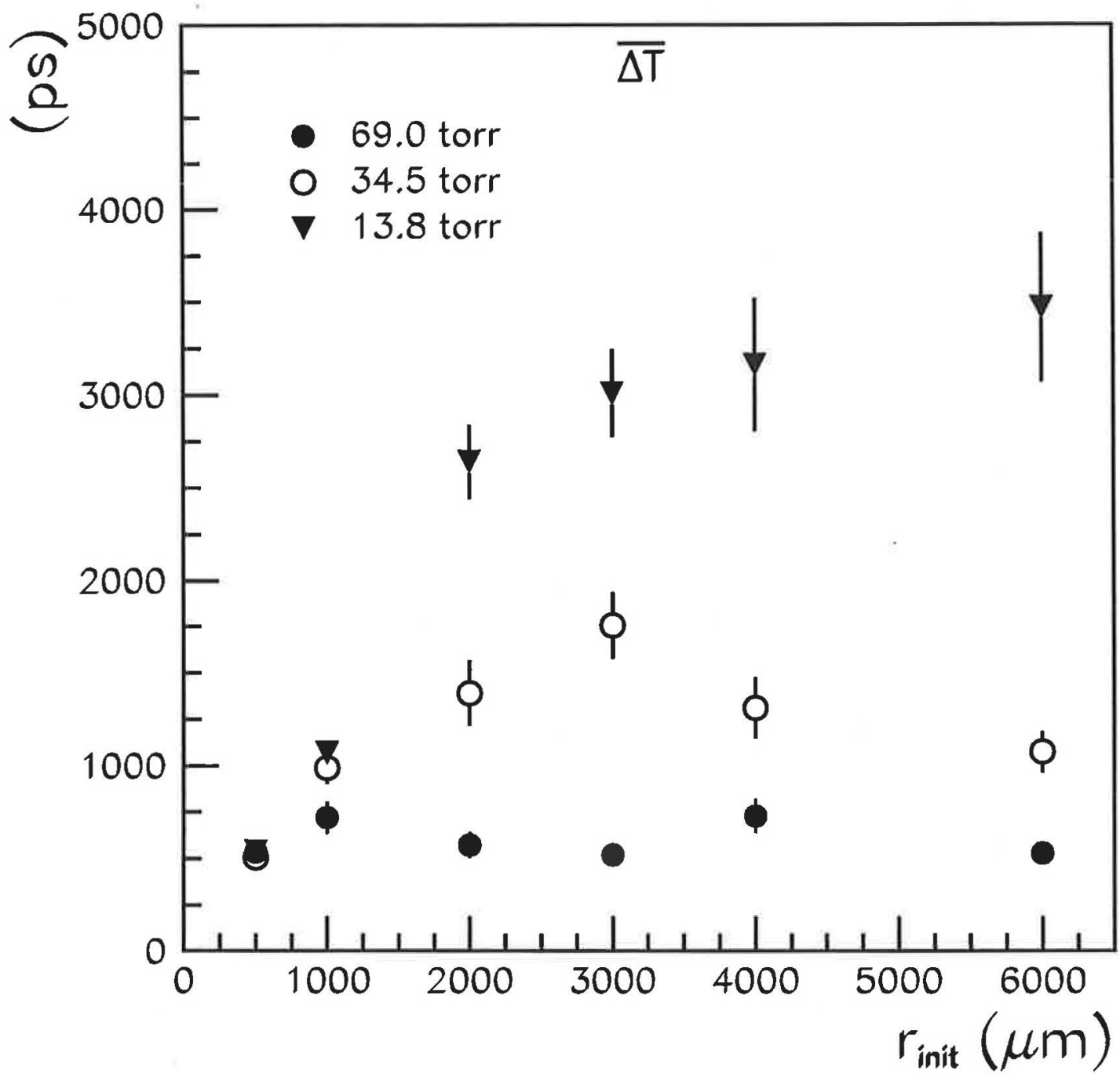

Figure 22: Dependence of the average time extent of avalanche $\overline{\Delta T}$ on the distance $r_{\text {init }}$ from the anode at which the primary electron was released. Calculated for 3 different gas pressures and anode voltage $700 \mathrm{~V}$. 



